\title{
Efeito da Luz e da Temperatura na Germinação de Sementes de Cochlospermum vitifolium (Will.) Sprengel
}

\author{
Diego de Sousa Pereira ${ }^{1}$, Magnum de Sousa Pereira ${ }^{2}$, \\ Antonio Marcos Esmeraldo Bezerra ${ }^{3}$
}

\author{
${ }^{1}$ Laboratório de Análise de Sementes, Programa de Pós-graduação em Agronomia-Fitotecnia, \\ Departamento de Agricultura, Universidade Federal de Lavras - UFLA, Campus Universitário, Lavras/MG, Brasil \\ ${ }^{2}$ Programa de Pós-graduação em Agronomia-Solos e Nutrição de Plantas, Departamento de Solos, \\ Universidade Federal do Ceará - UFC, Campus do Pici, Fortaleza/CE, Brasil \\ ${ }^{3}$ Programa de Pós-graduação em Agronomia-Fitotecnia, Departamento de Fitotecnia, \\ Universidade Federal do Ceará - UFC, Campus do Pici, Fortaleza/CE, Brasil
}

\begin{abstract}
RESUMO
Para avaliar a influência da luz e da temperatura na germinação de sementes de Cochlospermum vitifolium, frutos maduros foram coletados de 12 plantas matrizes. Os tratamentos foram mantidos em câmaras de germinação equipadas com lâmpadas fluorescentes brancas, num arranjo fatorial $2 \times 4$, constituídos por duas condições de luminosidade, com fotoperíodo de 8 $\mathrm{h}$ de luz e $16 \mathrm{~h}$ de escuro, e escuro completo, distribuídos em quatro regimes de temperatura, sendo três temperaturas constantes $\left(20,25\right.$ e $\left.30^{\circ} \mathrm{C}\right)$ e uma temperatura alternada $\left(20-30^{\circ} \mathrm{C}\right)$, utilizando-se um modelo inteiramente casualizado, com quatro repetições de 50 sementes. Efetuaram-se contagens diárias até o $14 .^{\circ}$ dia após a semeadura, para avaliação da porcentagem de germinação, do índice de velocidade e do tempo médio de germinação. Conclui-se que as sementes de Cochlospermum vitifolium são fotoblásticas neutras e a temperatura constante de $20{ }^{\circ} \mathrm{C}$ é a condição mais adequada para a germinação da espécie.
\end{abstract}

Palavras-chave: Cochlospermaceae, ecofisiologia, vigor.

\section{Effect of Light and Temperature on Seed Germination of Cochlospermum vitifolium (Will.) Sprengel}

\begin{abstract}
In this study, we aimed to evaluate the influence of light and temperature on the germination of Cochlospermum vitifolium seeds. To this end, ripe fruits were picked from twelve mother plants and the treatments were kept in a germination chamber equipped with white fluorescent lamps in a $2 \times 4$ factorial arrangement consisting of two light conditions, with a photoperiod of $8 \mathrm{~h}$ of light and $16 \mathrm{~h}$ of darkness and total darkness, divided in four temperature regimes: three constant temperatures $\left(20,25\right.$ and $\left.30^{\circ} \mathrm{C}\right)$ and an alternating temperature $\left(20-30{ }^{\circ} \mathrm{C}\right)$, using a completely randomized design with four replications of 50 seeds. Daily counts were performed until the 14th day after planting to evaluate the percentage of germination, speed rate, and average time of germination. We concluded that the seeds of Cochlospermum vitifolium are neutral photoblastic and the constant temperature of $20{ }^{\circ} \mathrm{C}$ is the best condition for the germination of this species.
\end{abstract}

Keywords: Cochlospermaceae, ecophysiology, vigor. 


\section{INTRODUÇÃO}

A família Cochlospermaceae compreende apenas dois gêneros, Cochlospermum e Amoreuxia, os quais reúnem cerca de 15 espécies distribuídas nas regiões tropicais, especialmente nas Américas e na África. Cochlospermum vitifolium (Will.) Sprengel. é uma pequena árvore, popularmente conhecida como algodão-bravo ou algodão-do-campo em diversos Estados do país, e como pacotê, no Ceará (Maia, 2004). A floração de C. vitifolium coincide com o período da desfolha, de dezembro a março, no hemisfério Norte, e de junho a outubro, abaixo da linha do equador (Poppendieck, 1981). Durante esse período, encontra-se totalmente despida de folhagem; entretanto, sua flor grande e vistosa, em cachos terminais de coloração amarela, destaca-se por fornecer pólen para abelhas (Agostini \& Sazima, 2003; Maia, 2004).

Em decorrência das múltiplas utilizações conferidas às espécies nativas, seja madeireira, apícola, forrageira ou medicinal, como o pacotê (Maia, 2004; Almeida et al., 2005), essas espécies vêm sofrendo vários processos de exploração intensa, de forma predatória, causando a devastação de suas populações naturais. Os conhecimentos técnicos, biológicos e ecológicos acerca da silvicultura de espécies florestais são imprescindíveis para a sua indicação nas ações inerentes ao estabelecimento de plantações florestais, particularmente em áreas de reconstituição (Rodrigues et al., 2007).

O acompanhamento da germinação de sementes é um dos aspectos ecofisiológicos mais estudados; além das características morfológicas, há a preocupação com relação à propagação dessas espécies, pois espécies nativas muitas vezes possuem seu processo germinativo limitado, em razão da dormência das sementes, que pode ser causada por impermeabilidade do tegumento a água e a gases; exigências especiais de luz e temperatura; imaturidade do embrião, e fatores fisiológicos inibitórios (Kucera et al., 2005; Finch-Savage \& Leubner-Metzger, 2006; Thompson \& Ooi, 2010).

A sensibilidade das sementes à luz é bastante variável de acordo com a espécie, havendo sementes cuja germinação é influenciada positiva ou negativamente pela luz e sementes indiferentes a esse fator, denominadas fotoblásticas positiva, negativa e neutra, respectivamente (Silva et al., 2002; Guedes et al., 2010; Guedes \& Alves, 2011).

Esse requerimento de luz para a germinação das sementes, em algumas espécies, é fortemente influenciado pela temperatura. Segundo Bewley \& Black (1994), é importante a determinação das temperaturas mínima, ótima e máxima para a germinação das espécies, pois a temperatura ótima propicia a máxima porcentagem de germinação em menor espaço de tempo, enquanto em temperaturas máximas e mínimas, as sementes perdem tal capacidade. De acordo com os autores, a temperatura pode afetar a absorção de água e as reações bioquímicas que regulam o metabolismo envolvido nesse processo, sendo a faixa de temperatura ótima característica de cada espécie.

A influência da temperatura e do fotoperíodo na germinação de sementes florestais é encontrada na literatura para diferentes espécies, como em Myracrodruon urundeuva Allemão (Silva et al., 2002), Caesalpinia echinata Lam. (Mello \& Barbedo, 2007), Dyckia tuberosa (Vell.) Beer (Vieira et al., 2007), Bowdichia virgilioides Kunth. (Albuquerque \& Guimarães, 2007), Heliocarpus popayanensis L. (Brancalion et al., 2008), Plantago tomentosa Lam. (Dousseau et al., 2008), Tabebuia roseo-alba (Ridl.) Sand. (Stockman et al., 2007), Cereus jamacaru DC. (Guedes et al., 2009), Dyckia goehringii Gross \& Rauh (Duarte et al., 2010), Amburana cearensis (Allemão) A.C. Smith (Guedes et al., 2010) e Chorisia glaziovii (O. Kuntze) (Guedes \& Alves, 2011).

Diante do exposto, objetivou-se avaliar a influência da luz e da temperatura na germinação de sementes de Cochlospermum vitifolium.

\section{MATERIAL E MÉTODOS}

Frutos maduros de pacotê foram colhidos de 12 plantas matrizes em Fortaleza-CE $\left(03^{\circ} 47^{\prime} 25^{\prime \prime} \mathrm{S}\right.$ e $38^{\circ}$ $\left.30^{\prime} 30^{\prime \prime} \mathrm{W}\right)$. No beneficiamento, as sementes foram extraídas manualmente, com a retirada da pluma aderida ao tegumento das sementes. Em seguida, selecionaram-se sementes sadias, sem a presença de patógenos ou atacadas por insetos.

Após o beneficiamento, determinou-se o teor de água das sementes, pelo método da estufa 
$\left(105 \pm 3^{\circ} \mathrm{C}\right)$ por 24 horas, com duas repetições de 50 sementes, além do peso de mil sementes, com oito repetições de cem sementes (Brasil, 2009).

Para a realização do experimento, as sementes foram submetidas à escarificação química com ácido sulfúrico (98\%) por 25 minutos, a fim de promover a quebra da dormência presente na espécie. Posteriormente, as sementes foram dispostas em placas de Petri com nove centímetros de diâmetro, contendo duas folhas de papel de filtro umedecidas com água destilada na proporção de 2,5 vezes o peso do papel seco. As placas foram mantidas em câmaras de germinação (B.O.D.) equipadas com lâmpadas fluorescentes brancas.

O delineamento experimental utilizado foi $\mathrm{o}$ inteiramente casualizado, num arranjo fatorial $2 \times$ 4, constituído por duas condições de luminosidade: fotoperíodo de $8 \mathrm{~h}$ de luz e 16 h de escuro, e escuro completo, e quatro regimes de temperatura: 20, 25, 20-30 e $30{ }^{\circ} \mathrm{C}$. Utilizaram-se quatro repetições de 50 sementes para cada tratamento.

Efetuaram-se contagens diárias do número de plântulas normais até o $14 .^{\circ}$ dia após a semeadura, para avaliação da porcentagem de germinação (PG), do índice de velocidade (IVG) (Maguire, 1962) e do tempo médio de germinação $(\bar{t})$ (Labouriau, 1983 apud Ferreira \& Borghetti, 2004). As sementes mantidas em escuro completo foram observadas sob luz verde.
Os dados foram submetidos à análise de variância e as médias comparadas pelo teste de Tukey em nível de 5\% de probabilidade, usando o aplicativo computacional SISVAR (Ferreira, 2003).

\section{RESULTADOS E DISCUSSÃO}

O peso médio de mil sementes da amostra em estudo foi de $26,33 \mathrm{~g}$ e o teor médio de água foi de $12,24 \%$.

Os resultados referentes à porcentagem de germinação, computados no $14 .^{\circ}$ dia após a semeadura, não revelaram interação significativa entre os fatores luminosidade e temperatura. Houve interação para os resultados referentes ao índice de velocidade de germinação. Entretanto, a temperatura influenciou significativamente o tempo médio, em que o efeito isolado foi mais evidenciado (Tabela 1).

Na avaliação da porcentagem de germinação, não foi observada diferença estatística entre as condições de luminosidade e os regimes de temperatura avaliados. A porcentagem de germinação média das sementes mantidas no escuro e das sementes submetidas ao fotoperíodo de oito horas de luz foi $73 \%$ e $71 \%$, respectivamente (Tabela 2 ).

Dessa forma, a incidência de luz não é um fator limitante para que as sementes de C. vitifolium iniciem o processo germinativo. Assim, as sementes

Tabela 1. Resumo da análise de variância e coeficiente de variações referentes a porcentagem (PG), índice de velocidade (IVG) e tempo médio de germinação $(\bar{t})$, obtidos em sementes de Cochlospermum vitifolium submetidas a diferentes condições de luz e temperatura.

Table 1. Summary of the variance analysis and coefficient of variation concerning the percentage (PG), speed rate (SRG) and average germination time $(\bar{t})$, obtained in Cochlospermum vitifolium seeds submitted to different conditions of light and temperature.

\begin{tabular}{ccccc|}
\hline \multirow{2}{*}{ FV } & GL & \multicolumn{3}{c|}{ Quadrados médios e significância } \\
\cline { 3 - 5 } & & PG & IVG & $\bar{t}$ \\
\hline Luz $(\mathrm{L})$ & 1 & $32,00^{\mathrm{ns}}$ & $3,49^{\mathrm{ns}}$ & $1,74^{\mathrm{ns}}$ \\
\hline Temperatura (T) & 3 & $41,33^{\mathrm{ns}}$ & $15,57^{\star *}$ & $1,85^{\star}$ \\
\hline L $\times$ T & 3 & $78,66^{\mathrm{ns}}$ & $7,47^{\star}$ & $0,48^{\text {ns }}$ \\
\hline Tratamentos & 7 & $56,00^{\mathrm{ns}}$ & $10,37^{\star *}$ & $1,25^{\mathrm{ns}}$ \\
\hline Erro & 24 & 58,00 & 2,15 & 0,62 \\
\hline Total & 31 & - & - & - \\
\hline CV $(\%)$ & - & 10,5 & 18,56 & 22,18 \\
\hline
\end{tabular}

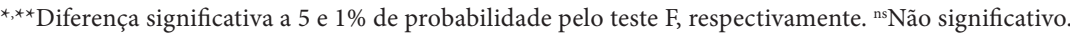


Tabela 2. Porcentagem de germinação de sementes de Cochlospermum vitifolium submetidas a diferentes condições de luz (A) e temperatura (B).

Table 2. Percentage of germination of Cochlospermum vitifolium seeds submitted to different light (A) and temperature (B) conditions.

\begin{tabular}{cccccc}
\multirow{2}{*}{ Luz } & \multicolumn{2}{c}{ Temperatura } & \multirow{2}{*}{ Média } \\
\cline { 2 - 5 } & $\mathbf{2 0}{ }^{\circ} \mathbf{C}$ & $\mathbf{2 5}{ }^{\circ} \mathbf{C}$ & $\mathbf{3 0} \mathbf{C}$ & $\mathbf{2 0 - 3 0}{ }^{\circ} \mathbf{C}$ & $73 \mathrm{~A}$ \\
Sem & 79 & 76 & 69 & 70 & $71 \mathrm{~A}$ \\
Com & 70 & 72 & 75 & 69 & $69 \mathrm{a}$ \\
\hline Média & $74 \mathrm{a}$ & $74 \mathrm{a}$ & $72 \mathrm{a}$ & \\
\hline
\end{tabular}

Médias seguidas de mesma letra, maiúscula na coluna e minúscula na linha, não diferem entre si pelo teste de Tukey a $5 \%$ de probabilidade $\left(\mathrm{DMS}_{\text {luz }}=5,55 ; \mathrm{DMS}_{\text {temp. }}=10,50\right)$.

podem ser classificadas como fotoblásticas neutras ou indiferentes à luz.

Resultados equivalentes foram obtidos por Silva et al. (2002) com sementes de aroeira (Myracrodruon urundeuva Allemão), por Biruel et al. (2007) com sementes de pau-ferro [Caesalpinia leiostachya (Benth.) Ducke] e por Brancalion et al. (2008) com sementes de jangada-brava (Heliocarpus popayanensis L.), em que as temperaturas ótimas de germinação dessas espécies ocorrem entre 20 e 30 ${ }^{\circ} \mathrm{C}$, independentemente da presença ou da ausência de luz.

Alguns autores relatam o efeito negativo de altas temperaturas $\left(35^{\circ} \mathrm{C}\right)$ na germinação de sementes; como exemplos, citam-se os resultados obtidos por Albuquerque \& Guimarães (2007) com sementes de sucupira-preta (Bowdichia virgilioides Kunth.) e por Guedes \& Alves (2011) em sementes de barriguda (Chorisia glaziovii O. Kuntze), em que temperaturas elevadas reduziram drasticamente a germinação das espécies.

Em outros casos, temperaturas acima de $30{ }^{\circ} \mathrm{C}$ podem representar um estímulo à germinação, como observado por Guedes et al. (2010) para sementes de cumaru [Amburana cearensis (Allemão) A.C. Smith], sendo, para essa espécie, a temperatura de $35^{\circ} \mathrm{C}$ a mais recomendada para se avaliar o processo germinativo das sementes.

Para a espécie em estudo, as temperaturas constantes proporcionaram, de forma quantitativa, as maiores médias de germinação, em comparação com a temperatura alternada $\left(20-30^{\circ} \mathrm{C}\right)$. Entretanto, o fato de ocorrer germinação em ambos os regimes de temperatura revela a capacidade da espécie de se adaptar às flutuações térmicas naturais do ambiente onde está inserida. Essa característica, segundo Guedes et al. (2010), confere às espécies, como Cochlospermum vitifolium, uma maior capacidade de estabelecimento, tornando-as mais resistentes às adversidades do ambiente, como as encontradas na região semiárida do nordeste brasileiro.

Para o índice de velocidade de germinação, observou-se que a combinação de luz e temperatura influenciou significativamente a velocidade de germinação das sementes avaliadas (Tabela 3 ).

As sementes germinadas no escuro, à temperatura de $25^{\circ} \mathrm{C}$, tiveram o índice de velocidade $(9,08)$ superior ao das sementes germinadas na mesma temperatura com presença da luz $(6,17)$. Na ausência de luz, nas temperaturas fixas de 20 e $25^{\circ} \mathrm{C}$, e alternada $20-30{ }^{\circ} \mathrm{C}$, apresentaram-se os maiores índices de velocidade de germinação.

Na presença de luz, é observado um maior índice de velocidade de germinação para a temperatura fixa de $20^{\circ} \mathrm{C}$, sendo superior ao observado para as temperaturas fixas de $25^{\circ} \mathrm{C}$ e alternada $20-30^{\circ} \mathrm{C}$.

Resultados diferentes foram observados por Albuquerque \& Guimarães (2007) para sementes de Bowdichia virgilioides(Kunth.). Os autores observaram, para temperatura alternada $\left(20-30{ }^{\circ} \mathrm{C}\right)$, uma superioridade para o índice de velocidade de germinação em relação às outras temperaturas, que são semelhantes às utilizadas neste ensaio.

Em sementes de Chorisia glaziovii (O. Kuntze), Guedes \& Alves (2011) observaram que os maiores índices de velocidade de germinação ocorreram na temperatura de $25{ }^{\circ} \mathrm{C}$. Em sementes de Plantago tomentosa (Lam.), Dousseau et al. (2008) observaram, pelo índice de velocidade, que as temperaturas de 15,20 e $15-25^{\circ} \mathrm{C}$ favoreceram a germinação, e que 
Tabela 3. Índice de velocidade de germinação de sementes de Cochlospermum vitifolium submetidas a diferentes condições de luz e temperatura.

Table 3. Germination speed rate of Cochlospermum vitifolium seeds submitted to different light and temperature conditions.

\begin{tabular}{cccccc}
\multicolumn{5}{c}{ Índice de Velocidade de Germinação } & \multirow{2}{*}{ Média } \\
\cline { 2 - 5 } Luz & $\mathbf{2 0}{ }^{\circ} \mathbf{C}$ & $\mathbf{2 5}{ }^{\circ} \mathbf{C}$ & $\mathbf{3 0}{ }^{\circ} \mathbf{C}$ & $\mathbf{2 0 - 3 0}{ }^{\circ} \mathbf{C}$ & \\
\cline { 2 - 5 } Sem & $9,78 \mathrm{Aa}$ & $9,08 \mathrm{Aab}$ & $6,37 \mathrm{Ab}$ & $7,62 \mathrm{Aab}$ & 7,21 \\
Com & $10,06 \mathrm{Aa}$ & $6,17 \mathrm{Bb}$ & $7,85 \mathrm{Aab}$ & $6,13 \mathrm{Ab}$ & 7,55 \\
Média & 9,92 & 7,62 & 7,11 & 6,88 & \\
\hline
\end{tabular}

Médias seguidas de mesma letra, maiúsculas nas colunas e minúsculas nas linhas, não diferem entre si pelo teste de Tukey a $1 \%$ de probabilidade $\left(\mathrm{DMS}_{\mathrm{T}: \mathrm{L}}=2,13 ; \mathrm{DMS}_{\mathrm{L}: \mathrm{T}}=2,85\right)$.

Tabela 4. Tempo médio de germinação de sementes de Cochlospermum vitifolium submetidas a diferentes condições de luz (A) e temperatura (B).

Table 4. Average time of germination of Cochlospermum vitifolium seeds submitted to different light (A) and temperature (B) conditions.

\begin{tabular}{cccccc}
\multirow{2}{*}{ Luz } & \multicolumn{3}{c}{ Temperatura } & \multirow{2}{*}{ Média } \\
\cline { 2 - 4 } & $\mathbf{2 0}{ }^{\circ} \mathbf{C}$ & $\mathbf{2 5}{ }^{\circ} \mathbf{C}$ & $\mathbf{3 0}{ }^{\circ} \mathbf{C}$ & $\mathbf{2 0 - 3 0}{ }^{\circ} \mathbf{C}$ & $3,29 \mathrm{~A}$ \\
Sem & 2,66 & 3,48 & 3,86 & 3,18 & $3,76 \mathrm{~A}$ \\
Com & 3,03 & 4,39 & 3,68 & 3,93 & \\
\hline Média & $2,84 \mathrm{~b}$ & $3,93 \mathrm{a}$ & $3,77 \mathrm{a}$ & $3,55 \mathrm{a}$ & \\
\hline
\end{tabular}

Médias seguidas de mesma letra, maiúsculas na coluna e minúsculas na linha, não diferem entre si pelo teste de Tukey a $5 \%$ de probabilidade $\left(\mathrm{DMS}_{\mathrm{luz}}=0,57 ; \mathrm{DMS}_{\text {temp. }}=0,70\right)$.

temperaturas acima de $25^{\circ} \mathrm{C}$ causaram prejuízos na porcentagem e na velocidade de germinação das sementes.

Não houve, para o tempo médio de germinação, interação significativa entre as diferentes temperaturas e as condições luminosas avaliadas. No entanto, o fator temperatura, em separado, influenciou significativamente o tempo médio de germinação (Tabela 4).

Para as sementes mantidas no escuro, o tempo médio de germinação observado foi de 3,29 dias, enquanto que as sementes mantidas em fotoperíodo de oito horas de luz apresentaram tempo médio de 3,76 dias. A temperatura fixa de $20{ }^{\circ} \mathrm{C}$ foi a que melhor favoreceu a germinação das sementes de C. vitifolium, dado verificado pelo menor tempo médio de germinação. O comportamento germinativo observado, neste estudo, de sementes de Cochlospermum vitifolium, diverge do observado para sementes de Licania rigida (Benth), que é uma espécie fotoblástica neutra, cujo tempo médio de germinação reduziu-se com o aumento da temperatura, sendo as temperaturas de 25 e $30^{\circ} \mathrm{C}$ as que proporcionaram os melhores resultados (Diniz et al., 2008).

\section{CONCLUSÕES}

Sementes de Cochlospermum vitifolium são fotoblásticas neutras.

A temperatura constante de $20{ }^{\circ} \mathrm{C}$ é a condição mais favorável para a germinação de sementes de $C$. vitifolium.

\section{STATUS DA SUBMISSÃO}

Recebido: 27/02/2013

Aceito: $24 / 07 / 2013$

Publicado: 30/09/2013

\section{AUTOR(ES) PARA CORRESPONDÊNCIA}

\section{Diego de Sousa Pereira}

Laboratório de Análise de Sementes, Programa de Pós-graduação em AgronomiaFitotecnia, Departamento de Agricultura, Universidade Federal de Lavras - UFLA, Campus Universitário, CP 3037, CEP 37200-000, Lavras, MG, Brasil

e-mail: diegobizi@gmail.com 


\section{APOIO FINANCEIRO}

\section{$\mathrm{CNPq} / \mathrm{UFC}$}

\section{REFERENNCIAS}

Agostini K, Sazima M. Plantas ornamentais e seus recursos para abelhas no Campus da Universidade Estadual de Campinas, Estado de São Paulo, Brasil. Bragantia 2003; 62(3): 335-343. http://dx.doi. org/10.1590/S0006-87052003000300001

Albuquerque KS, Guimarães RM. Comportamento fisiológico de sementes de Bowdichia virgilioides Kunth. sob diferentes condições de luz e temperaturas. Cerne 2007; 13(1): 64-70.

Almeida SCX, Lemos TLG, Silveira ER, Pessoa ODL. Constituintes químicos voláteis e não-voláteis de Cochlospermum vitifolium (WILLDENOW) SPRENGEL. Química Nova 2005; 28(1): 57-60. http:// dx.doi.org/10.1590/S0100-40422005000100012

Bewley JD, Black M. Seeds: physiology of development and germination. New York: Plemun Press; 1994. 445 p.

Biruel RP, Aguiar IB, Paula RC. Germinação de sementes de pau-ferro submetidas a diferentescondições de armazenamento, escarificação química, temperatura e luz. Revista Brasileira de Sementes 2007; 29(3): 151-159. http://dx.doi.org/10.1590/S0101-31222007000300018

Brancalion PHS, Novembre ADLC, Rodrigues RR, Chamma HMCP. Efeito da luz e de diferentes temperaturas na germinação de sementes de Heliocarpus popayanensis L. Revista Árvore 2008; 32(2): 225-232. http://dx.doi.org/10.1590/S0100-67622008000200005

Brasil. Ministério da Agricultura e Reforma Agrária. Regras para análises de sementes. Brasília: SNDA/ DNDV/CLAV; 2009. 399 p.

Diniz FO, Moreira FJC, Silva FDB, Medeiros S Fo. Influência da luz e temperatura na germinação de sementes de oiticica (Licania rigida Benth.). Revista Ciência Agronômica 2008; 39(3): 476-480.

Dousseau S, Alvarenga AA, Arantes LO, Oliveira D, Nery FC. Germinação de sementes de tanchagem (Plantago tomentosa Lam.): influência da temperatura, luz e substrato. Ciência e Agrotecnologia 2008; 32(2): 438-443. http://dx.doi.org/10.1590/S141370542008000200014

Duarte EF, Carneiro IF, Silva NF, Guimarães NNR. Características físicas e germinação de sementes de Dyckia goehringii Gross \& Rauh (BROMELIACEAE) sob diferentes temperaturas. Pesquisa Agropecuária Tropical 2010; 40(4): 422-429. http://dx.doi.org/10.5216/pat. v40i4.6037
Ferreira DF. SISVAR - Sistemas de análises estatísticas. Lavras: UFLA; 2003.

Ferreira AG, Borghetti F, organizadores. Germinação: do básico ao aplicado. Porto Alegre: Artmed; 2004. p. 135-146.

Finch-Savage W, Leubner-Metzger W. Seed dormancy and the control of germination. New Phytologist 2006; 171(3): 501-523. PMid:16866955. http://dx.doi. org/10.1111/j.1469-8137.2006.01787.x

Guedes RS, Alves EU, Gonçalves EP, Bruno RLA, Braga JM Jr, Medeiros MS. Germinação de sementes de Cereus jamacaru DC.em diferentes substratos e temperaturas. Acta Scientiarum.Biological Sciences 2009; 31(2): 159164.

Guedes RS, Alves EU, Gonçalves EP, Braga JM Jr, Viana JS, Colares PNQ. Substratos e temperaturas para testes de germinação e vigor de sementes de Amburana cearensis (Allemão) A.C. Smith. Revista Árvore 2010; 34(1): 57-64. http://dx.doi.org/10.1590/S010067622010000100007

Guedes RS, Alves EU. Substratos e temperaturas para o teste de germinação de sementes de Chorisia glaziovii (O. Kuntze). Cerne 2011; 17(4): 525-531.

Kucera B, Cohn MA, Leubner-Metzger G. Plant hormone interactions during seed dormancy and germination. Seed Science Research 2005; 15(4): 281 307. http://dx.doi.org/10.1079/SSR2005218

Maia GN. Caatinga: árvores e arbustos e suas utilidades. São Paulo; 2004. 413 p.

Maguire JD. Speed of germination and selection and evaluation for seedling emergence and vigor. Crop Science 1962; 2(2): 176-177. http://dx.doi.org/10.2135/ cropsci1962.0011183X000200020033x

Mello JIO, Barbedo CJ. Temperatura, luz e substrato para germinação de sementes de pau-brasil (Caesalpinia echinata Lam., Leguminosae Caesalpinioideae). Revista Árvore 2007; 31(4): 645-655. http://dx.doi.org/10.1590/ S0100-67622007000400009

Poppendieck HH. Flora Neotrópica, Cochlospermaceae. New York: Organization for Flora Neotropica, The New York Botanical Garden; 1981.

Rodrigues PMS, Silva CHP, Braga LL, Nunes YRF, Veloso MDM, Gonzaga APD. Efeito da Luz e da Procedência na Germinação de Sementes de Copaifera langsdorffii Desf. (Fabaceae - Caesalpinoideae). Revista Brasileira de Biociências 2007; 5(2): 264-266.

Silva LMM, Rodrigues TJD, Aguiar IB. Efeito da luz e da temperatura na germinação de sementes de aroeira (Myracrodruon urundeuva Allemão). Revista Árvore 2002; 26(6): 691-697. http://dx.doi.org/10.1590/S010067622002000600006 
Stockman AL, Brancalion PHS, Novembre ADLC, Chamma HMCP. Sementes de ipê-branco (Tabebuia roseo-alba (Ridl.) Sand. - Bignoniaceae): temperatura e substrato para o teste de germinação. Revista Brasileira de Sementes 2007; 29(3): 139-143. http://dx.doi. org/10.1590/S0101-31222007000300016

Thompson K, Ooi MKJ. To germinate or not to germinate: more than just a question of dormancy. Seed
Science Research 2010; 20(4): 209-211. http://dx.doi. org/10.1017/S0960258510000267

Vieira DCM, Socolowski F, Takaki M. Germinação de sementes de Dyckia tuberosa (Vell.) Beer (Bromeliaceae) sob diferentes temperaturas em luz e escuro. Revista Brasileira de Botânica 2007; 30(2): 183-188. http:// dx.doi.org/10.1590/S0100-84042007000200003 